\title{
Perícias Psicológicas em Casos de Conflito Interparental: Recomendações para a Prática
}

\author{
Ana Isabel Sani ${ }^{1}$ \\ Faculdade de Ciências Humanas e Sociais, Universidade Fernando Pessoa, Porto, Portugal
}

\section{Resumo}

A avaliação psicológica forense traduz-se frequentemente num trabalho de assessoria técnica aos tribunais, em matérias de particular complexidade e em domínios diversos, às quais a psicologia poderá dar especial contributo explicativo. A realização de perícias psicológicas em processos judiciais que envolvem crianças em situações difíceis de conflitos interparentais exige da parte dos técnicos conhecimentos e competências especiais, de modo a melhor responder à solicitação judiciária considerando o superior interesse da criança. Este artigo foca alguns dos principais aspectos que devem ser analisados, encetando uma reflexão sobre as áreas e os elementos que devem ser atendidos e/ou privilegiados nessa avaliação e que nos remetem, entre outros, para uma série de subjetividades e explorações que ultrapassam o domínio do discurso e dos fatos. As dinâmicas familiares, as relações e vinculações entre os membros da família, os afetos e desafetos exprimidos são dimensões que devem ser analisadas, para os quais existem subjacentes processos simples e complexos. A perícia psicológica forense deve reunir a análise compreensiva de todos estes aspectos, combinando o conhecimento teórico com a competência técnica e científica para o melhor interesse da criança.

Palavras-chave: Avaliação psicológica, tribunais, conflitos interparentais, perícia, criança.

\section{Psychological Expertise in Cases of Interparental Conflict: Recommendations for Practice}

\begin{abstract}
Forensic psychological assessment often comprises technical guidance in courts, especially in more complex matters in different areas to which psychology can provide significant explanatory support. Psychological examination in court proceedings involving children in difficult situations of parenting conflicts requires expert technicians to have specific knowledge and competence to efficiently respond to judicial demands, taking into account the best interest of the child. This article reviews some of the key aspects to be analyzed, proposing a reflection on the areas and elements that should be considered and/or prioritized in such assessments and that raise a series of subjectivities and explorations that go beyond the domain of discourse and facts. The family dynamics, relationships and bonds between family members, expressed preferences and dislikes are dimensions that must to be analyzed for which there simple and complex underlying processes. The forensic psychological assessment should bring together

Endereço para correspondência: Universidade Fernando Pessoa, Faculdade de Ciências Humanas e Sociais, Praça 9 de abril, 349, Porto, Portugal 4249-004. Fone: + 351225071300 | ext. 2305; Fax: +351 22 5508269; E-mail: anasani@ufp.edu.pt

Este trabalho foi financiado por Fundos Nacionais através da FCT (Fundação para a Ciência e a Tecnologia) e cofinanciado pelo Fundo Europeu de Desenvolvimento Regional (FEDER) através do COMPETE 2020 - Programa Operacional Competitividade e Internacionalização (POCI) no âmbito do CIEC (Centro de Investigação em Estudos da Criança da Universidade do Minho) com a referência POCI-01-0145FEDER-007562.
\end{abstract}


the comprehensive analysis of all these aspects, combining theoretical knowledge with technical and scientific competence for the best interests of the child.

Keywords: Psychological assessment, courts, interparental conflicts, expertise, child.

\section{Pericia Psicológica en Casos de Conflicto entre los Padres: Recomendaciones para la Práctica}

\section{Resumen}

La evaluación psicológica forense consiste frecuentemente en una actividad de asesoría técnica a los tribunales en materias de especial complejidad y en diversos ámbitos en que la psicología puede realizar aportaciones explicativas del caso en análisis. La realización de pericias psicológicas en procesos judiciales que implican los niños ante un conflicto entre padres requiere, por parte de los técnicos, conocimientos y habilidades especiales para que la decisión judicial tenga en consideración el interés superior del menor. Este artículo se centra en algunos de los aspectos que deben ser analizados en la evaluación psicológica, haciéndonos una reflexión sobre los elementos que debe recoger esa evaluación y que nos conducen a abordar una serie de subjetividades y exploraciones que van más allá del análisis del discurso y de los hechos. La dinámica familiar, las relaciones y vínculos entre los miembros de la familia, los afectos y desafectos expresados son dimensiones que deberán ser examinadas, para los cuales existen subyacentes procesos simples y complejos. La pericia psicológica forense debe cumplir con el análisis exhaustivo de todos estos aspectos, combinando los conocimientos teóricos con la experiencia técnica y científica para el mejor interés del niño.

Palabras clave: Evaluación psicológica, tribunales, conflictos entre los padres, pericia, niños.

A família detém um importante papel na socialização da criança. Os pais e cuidadores são os modelos de referência privilegiados que moldam na criança atitudes e comportamentos, que mais tarde esta poderá reproduzir nos seus relacionamentos (Bevan \& Higgins, 2002). Por essa razão é fundamental que o desenvolvimento da criança se processe de forma equilibrada e harmoniosa. Porém, se os relacionamentos na família forem marcados por conflitos violentos é possível que a criança apreenda e reproduza, no seio desta e noutros contextos, as interações aversivas que observou (Laporte, Jiang, Pepler, \& Chamberland, 2011). A observação de comportamentos violentos por parte dos jovens reforça a ideia de que é legítimo viver em conflito (Cottrell \& Monk, 2004), criando condições para a transmissão intergeracional da violência (Almeida \& Sani, 2014; Black, Sussman, \& Unger, 2010; Marasca, Colossi, \& Falcke, 2013).

A literatura científica (e.g., Dankoski et al., 2006; Fergusson, Boden, \& Horwood, 2008; Laporte et al., 2011; Simon \& Furman, 2010) tem vindo a identificar a relação positiva entre presenciar na família conflitos violentos e envolver-se em relações amorosas conflituosas no futuro. Assim sendo, a exposição à violência do casal, frequentemente, progenitores da criança (violência interparental), que podem estar unidos ou não por laços de conjugalidade representa um importante fator de risco de reprodução, no futuro, de dinâmicas relacionais violentas. Para além disso, estes conflitos violentos constituem igualmente uma forma de vitimação da criança que, embora indireta, tem implicações sérias no seu desenvolvimento equilibrado (Sani, 2011). A ocorrência dos conflitos violentos num espaço familiar, o qual tendemos a assumir como um local de proteção e o fato das pessoas envolvidas serem, por norma, figuras de vinculação e afeto, acaba também por ocasionar o impacto negativo dessa experiência (Sani \& Almeida, 2011a).

É no decurso de acompanhamentos e avaliações realizadas no âmbito de processos de promoção e proteção de crianças e jovens ou no contexto de divórcios litigiosos que muitos dos 
conflitos familiares violentos ganham visibilidade social, sendo reconhecidos vários dos aspectos suprarreferidos, os quais são aprofundadamente estudados e salientados de modo a apoiar a tomada de decisão judicial. A assessoria técnica no âmbito destes processos pode constituir-se como uma tarefa particularmente complexa e exigente para os psicólogos forenses (Machado \& Sani, 2014). Estes profissionais devem estar muito bem munidos de conhecimento e competências técnicas e pessoais capazes de exprimir de forma isenta os contornos, vicissitudes e repercussões ocasionadas pela existência de conflitos interparentais.

Assim, a propomo-nos primeiramente fazer uma abordagem, assente numa revisão teórica, ainda que sucinta, à compreensão do impacto deste fenômeno de exposição da crianças aos conflitos interparentais, para posteriormente, explicitarmos os elementos cruciais na realização de uma perícia psicológica forense no âmbito de processos que envolvem crianças que tenham experienciado este tipo de fenômeno.

\section{Os Efeitos da Exposição aos Conflitos Interparentais nas Crianças}

Alguns estudos (e.g., English et al., 2009; Herrenhohl, Sousa, Tajima, Herrenhohl, \& Moylan, 2008) referem que a exposição das crianças aos conflitos violentos entre os pais é mais comum do que os maus tratos à criança. $\mathrm{O}$ fato é que mesmo não reconhecendo por completo a abrangência estatística do fenômeno em Portugal, os dados de investigação no país (cf. Sani \& Almeida, 2011a) têm vindo a alertar para os efeitos que a exposição à violência interparental poderá ter no ajustamento global da criança. As consequências para crianças são sobretudo a nível psicológico (Sani, 2011), embora se detectem problemas clinicamente significativos em várias níveis (fisiológico, emocional, cognitivo, comportamental; Coutinho \& Sani, 2008; Patias, Bossi, \& Dell'Aglio, 2014; Sani, 2007).

Os conflitos interparentais podem assumir diferentes níveis de conflitualidade que têm implicações distintas no exercício da parentalidade materna e paterna e, consequentemente, o estresse parental gerado interfere com o ajustamento da criança. Num estudo realizado por Camisasca, Miragoli e Di Blasio (2016) com 358 pares (pai e mãe) de crianças em idade escolar, os autores demonstraram que níveis elevados de conflito interparental afetam negativamente a adaptação da criança. No entanto mesmo os conflitos menos abertos ou evidentes têm um impacto negativo na criança. $O$ fato de os pais não falarem acerca dos seus problemas e não assumirem a existência do conflito quando este é iminente, gera na criança uma grande angústia e confusão, pois esta não está capaz de compreender os relacionamentos. Não raras vezes a criança sente que deve fazer algo para resolver a situação, porém nem sempre o consegue (Pryor \& Pattison, 2007). Se as crianças assistirem a conflitos de maior intensidade, a perceção de controle é mais diminuta e tal contribui para um impacto negativo da experiência (Sani, 2011). Esse impacto poderá ser particularmente significativo se a criança tiver escassez de recursos pessoais para o confronto (Hughes, Graham-Bermann, \& Gruber, 2001), o que remete para a consideração necessária dos fatores mediadores do impacto dessa experiência.

De fato, o efeito que a exposição aos conflitos interparentais tem na criança vai depender de diversos fatores que podem estar relacionados com aspectos da criança, da família ou com as características dos incidentes que experienciam (Howell, 2011; Sani, 2006). As reações das crianças perante os conflitos interparentais são muito diversas, podendo algumas crianças exibir trauma ou graus sérios de desajustamento (Costa \& Sani, 2007) e outras leves indicadores ou mesmo aparente ausência sintomas. Isto revela que na análise que possamos fazer do impacto da exposição aos conflitos interparentais no ajustamento da criança seja necessário considerar de fatores de risco e de proteção associados à criança, aos pais e ao meio (Hughes et al., 2001; Sani, 2006). Assim, são exemplos de fatores mediadores certos atributos da criança, tais como a idade, o sexo, o estilo cognitivo, o nível de inteligência, a personalidade, o temperamento, as estratégias de 
coping, as perceções e interpretações da criança, assim como fatores mais de caráter situacional, como a experiência prévia em lidar com eventos stressantes (Cunningham \& Baker, 2004; Sani, 2006) e o sucesso no confronto com os mesmos. Outros fatores mais ligados à família são também referenciados como o estatuto socioeconómico, o estilo educativo dos pais (Sani, 2006), a sensibilidade da mãe (Levendosky, Bogat, \& HuthBocks, 2011; Manning, Davies, \& Cicchetti, 2014), os relacionamentos com os irmãos (Dunn \& Davies, 2001), assim como o apoio positivo prestado pelo grupo de pares (Bowen, 2015). Os fatores contextuais mais relacionados com o conflito (e.g., local de ocorrência, frequência, intensidade, duração, conteúdo, resolução do conflito) apoiam a própria construção que a criança faz sobre a gravidade dos episódios violentos (Sani, 2011) e como tal medeiam igualmente o impacto da experiência.

A investigação e a prática clínica (Sani, 2004a) têm vindo a certificar a existência de um conjunto de mecanismos diretos e indiretos (Hughes et al., 2001; Sani, 2006) capazes de influenciar o impacto na criança da exposição aos conflitos interparentais. Com base em alguns desses estudos emergiram diversas teorias explicativas que ajudam a compreender como pode processar-se esse impacto (cf. Sani, 2004b). Assim, apoiados neste conhecimento sobre o fenômeno somos levados a desenhar procedimentos científica, técnica e eticamente ajustáveis às exigências de uma avaliação pericial que responda à necessidade uma tomada de decisão judicial voltada ao superior interesse da criança.

\section{A Perícia Psicológica Forense em Casos de Conflitos Interparentais}

A realização de perícia psicológica para efeitos de decisão judiciária em casos que envolvem crianças expostas a conflitos interparentais exige da parte do técnico, como já antes afirmado, um conjunto de conhecimentos teóricos e práticos que lhe permitam organizar, de forma flexível e ajustada aos objetivos pretendidos, os procedimentos avaliativos (Sani \& Almeida, 2011b). Dado o caráter muitas vezes sensível destes casos, pela presença de crianças que poderão estar numa situação de risco, a avaliação não será alheia à necessidade de intervenção que preveja a proteção das mesmas.

As perícias psicológicas em casos de elevada conflitualidade familiar, que envolvem não apenas adultos, mas também crianças, são geralmente complexos e difíceis em termos de avaliação (Machado \& Sani, 2014; Pereira \& Matos, 2011). Estes casos têm sido objeto de frequentes pedidos por parte das entidades judiciais de pareceres psicológicos forenses. A complexidade das questões estão geralmente relacionadas com o risco que adultos e crianças podem enfrentar neste contexto, onde possam ser exibidos comportamentos violentos, direta ou indiretamente infligidos e, naturalmente com questões do exercício da parentalidade (Agulhas \& Anciães, 2014; Camisasca et al., 2016; Peixoto, Ribeiro, \& Manita, 2007; Pereira \& Matos, 2011). O risco não deixa também de estender-se aos profissionais envolvidos, existindo uma grande probabilidade de verem as suas práticas contestadas e refutadas através de queixas e processos (Machado \& Sani, 2014; Pereira \& Matos, 2011). Os relatórios periciais devem ser claros e credíveis e responder aos objetivos e quesitos formulados pelo Tribunal.

O processo pericial em psicologia envolve o recurso a múltiplas fontes (e.g., pais, criança, outros significativos), metodologias diversas (e.g., entrevistas, observação, aplicação de instrumentos, análise documental do processo e outros elementos relevantes) e a análise do problema nas suas variadas vertentes (e.g., dinâmicas familiares, parentalidade, práticas educativas, ajustamento global), para que o relatório produzido possa apoiar as decisões judiciais (Sani \& Almeida, 2011b). Em processos de regulação das responsabilidades parentais uma das solicitações judiciais comuns é a avaliação das competências parentais, a fim de aferir, sobretudo em casos de intenso litígio, qual dos progenitores revela maior capacidade $\mathrm{e}$ competência para assegurar o desenvolvimento integrado da criança (Agulhas, 2014; Peixoto et al., 2007). Naturalmente que muitos fatores 
revelam-se aqui de particular importância para a análise, designadamente por não haver um modelo universalmente aceito que defina como se exprime uma "parentalidade minimamente adequada" (Pereira \& Alarcão, 2010). O que se denota é que além da diversidade de modelos teóricos que abordam o conceito de parentalidade, focalizando vários aspectos, propondo múltiplos métodos e parâmetros de avaliação (Pereira \& Alarcão, 2010), o carácter probabilístico de qualquer avaliação deve tornar ciente que a avaliação das capacidades parentais não poderá nunca ser o único meio de prova a ser usado na decisão (Peixoto et al., 2007). Aliás numa situação em que a avaliação denota que ambos os pais revela capacidade parental adequada ao exercício da parentalidade, outros elementos são certamente chamados ao processo (e.g., avaliação psicológica das crianças, outros pareceres) de modo a apoiar as conclusões avançadas pela avaliação desses progenitores (Machado \& Sani, 2014; Peixoto et al., 2007). Desta forma, embora sem a pretensão de listar a totalidade de elementos cruciais na avaliação pericial em casos de conflitos interparentais, resulta imprescindível alguma sistematização de aspectos que poderão ser orientadores da prática forense neste tipo de perícias.

\section{Recomendações para uma Avaliação em Casos de Conflitos Interparentais}

Como em qualquer avaliação psicológica forense, a iniciação do caso pressupõe um conhecimento de quem solicita a perícia, dos quesitos formulados e das particularidades do caso, daí a necessidade de consultarmos os dados processuais que apoiam a compreensão do mesmo (Sani \& Almeida, 2011b). Esta consulta de dados do processo é, por norma, um elemento unânime nos diversos protocolos de avaliação, (e.g., Peixoto et al., 2007; Pereira \& Matos, 2011). Para além disso temos o consentimento informado e outras autorizações, obtidas também de início e que se revelam cruciais para o início das diversas outras fases do processo avaliativo. Em certos casos, o recurso à gravação das entrevistas faz destas solicitações elementos chave na prossecução da perícia.

Através da realização da perícia procurar-se-á, através de diversos meios e técnicas, dados clínicos e outros que permitam caracterizar a condição da criança e sua família, conhecer as dinâmicas familiares, os processos psicológicos e psicossociais compreendidos na parentalidade e a sua dimensão subjetiva (Peixoto et al., 2007). Assim serão avaliados aspectos como a qualidade dos vínculos emocionais entre a criança e os pais, a disponibilidade e a capacidade de um progenitor fomentar o relacionamento da criança com o outro, a participação na educação do filho, a ausência de situações de violência (cf. Huss, 2011). Tendo sempre presente os aspectos teóricos anteriormente debatidos sobre a inexistência de um quadro de referência universal sobre a parentalidade adequada, assim como as especificidades culturais, contextuais e histórico-temporais de modo flexível (Fialho, 2012; Peixoto et al., 2007), o relatório pericial deve apoiar a decisão judiciária, que terá sempre em consideração o superior interesse da criança. Vários organismos (e.g., American Academy of Child and Adolescent Psychiatry; American Psycological Association; Association of Family and Conciliation Courts) têm vindo a sugerir propostas que visam a uniformização dos procedimentos de avaliação, tomando por referência áreas preferenciais (Agulhas, 2014), como por exemplo qualidade do exercício do papel parental (e.g., as competências parentais, as práticas educativas) ou as dinâmicas relacionais (pais-filhos, na fratria, atitudes de cooperação, a comunicação entre progenitores).

A qualidade da parentalidade é um dos aspectos centrais de avaliação. A dissolução da relação conjugal dos progenitores não invalida que os mesmos, em questões de particular importância (e.g., escolha do estabelecimento de ensino, intervenções cirúrgicas, atividade laboral, orientação religiosa, entre outras) tenham de assumir em comum as responsabilidades parentais (cf. Fialho, 2012). Neste sentido importa perceber se essa cocoordenação parental é possível e se o exercício da parentalidade de cada um é funcional ou se, por seu turno, tal não se revela pra- 
ticável e fere os interesses e direitos da criança à proteção e desenvolvimentos integrais. Neste último caso, pode haver lugar a um exercício exclusivo das responsabilidades parentais, sendo os casos onde se comprova existirem conflitos interparentais violentos, um claro exemplo dessa possibilidade (Agulhas \& Anciães, 2014).

As dinâmicas familiares são outro dos elementos cruciais desta avaliação pericial. A existência de conflitos interparentais violentos nega a possibilidade de um desenvolvimento seguro e equilibrado de uma criança numa família, razão pela qual a convivência partilhada dos progenitores envolvidos em dinâmicas abusivas seja de todo de desconsiderar. Deste modo a eventual definição das responsabilidades parentais e/ou guarda exclusiva, naturalmente terá implicações no estabelecimento de relações pais - criança e na qualidade da mesma. A diminuição dos contatos filioparentais não favorece a vinculação entre eles, o que não é o que se pretende com esta determinação judicial. Porém, a literatura científica sustenta que a criança pode, mesmo vivendo mais separada de um ou outro progenitor, desenvolver laços seguros com ambos, o que pode permitir-lhe uma experienciação menos intensa e negativa dos conflitos interparentais (Cox, Paley, \& Harter, 2001). Isto só é possível se os progenitores, não obstante manterem o seu conflito conjugal, separarem-no das responsabilidades parentais que cada um têm de assumir.

$\mathrm{Se}$ as decisões geradas permitirem cessar esse contato da criança com os conflitos interparentais e se na distribuição de tempo que esta tem com os progenitores, possam vir a ser operadas condições que favoreçam a construção de laços (e não a destruição dos mesmos) será este um elemento importante a ser realçado na avaliação. Todavia se a avaliação despista uma vivência real negativa (Agulhas \& Anciães, 2014), por exemplo experiência direta ou indireta de violência na família, importa perceber se a rejeição manifestada é própria da criança (e.g., porque era vítima de mau trato) ou induzida por terceiros. Poderão existir processos psicológicos complexos que há necessidade de uma avaliação compreensiva, sob pena de se fazerem interpretações convencionais. Por exemplo, uma situação de rejeição da criança de um progenitor pode surgir por empenho do outro progenitor em afetar a relação da criança com aquele (tentativa de alienação parental ${ }^{2}$ ), como pode surgir da insuportabilidade para a criança em assistir à constante passividade de um progenitor ao conflito interparental, como da revolta contra o comportamento abusivo de um progenitor, como pode ser um estilo que foi sendo adotado pela criança como efeito direto da exposição aos conflitos interparentais ou resultar de um outro qualquer processo que seja importante aprofundar do ponto de vista da avaliação psicológica.

Realça-se a importância do perito $^{3}$ adotar uma avaliação dinâmica da família e perceber que processos podem estar a ser operados e quem os mobiliza. A avaliação da própria criança é aqui um elemento chave, considerando-se aspectos como o discurso (e.g., as justificações, as contradições, as pausas) ou a postura exibidos. Importa assim atender ao comportamento verbal e não-verbal e, sobretudo, avaliar como se apresentam, durante o período da avaliação, a todos os aspectos assinalados como relevantes.

2 Esta é uma forma de mau trato infantil com impacto negativo em várias dimensões da vida da criança (Machado \& Sani, 2015), sendo fundamental a identificação e sinalização precoce destes casos, devendo a intervenção, dependente dos níveis de alienação presentes, privilegiar uma abordagem multidisciplinar que se centre nos interesses e necessidades da criança e nas capacidades parentais para as satisfazer (Molinari \& Sani, 2015a, 2015b; Molinari \& Trindade, 2014).

3 Este tem definidas as suas funções no art. $153^{\circ}$ do Código Processo Penal português. Importa ainda referir que em Portugal não existe a figura do assistente técnico tal como descreve a legislação do Brasil. Em Portugal, a lei exclui (em regra) um regime de perícias adversariais que assente na possibilidade da parte no processo poder apresentar outra perícia que não a realizada pelo perito nomeado pelo tribunal (Gomes de Sousa, 2011). O Código Processo Penal português prevê, contudo, a figura do consultor técnico, que tendo funções de fiscalização e de assessor da parte, pode assistir à realização da perícia, com a possibilidade de intervir (art. 155. ${ }^{\circ}$, n. $^{\circ}$ 2) e de ser ouvido em audiência (art. $3500^{\circ}$ ), mas não de participar na elaboração do relatório pericial (art. 157. ${ }^{\circ}$ ). 
Neste sentido recomenda-se que todo o protocolo de avaliação que possa ser usado na realização de perícias psicológicas em casos de conflito interparental seja compreensivo e integrativo, envolvendo todo o sistema fami-liar e a triangulação de múltiplas fontes e metodologias, devendo ser suficientemente flexível para que possa ajustar-se a cada caso particular. Além dos procedimentos e técnicas já elencados (e.g., consulta do processo; consentimentos; entrevistas; observação de interações) recordamos que há uma série de técnicas específicas que podem igualmente apoiar a realização deste tipo de perícias. Assim a literatura internacional tem revelado especial consenso no uso de instrumentos empiricamente validados (e.g., Achenbach System of Empirically Based Assessment - ASEBA; Achenbach \& Rescorla, 2004; Achenbach et al., 2014), assim como no uso privilegiado da observação e de entrevistas semi-estruturadas, quer à criança (e.g., $\mathrm{Se}$ mistructured Clinical Interview for Children \& Adolescents - SCICA) quer aos adultos (individuais e coletivas), que permitam avaliar, entre muitos aspectos, as dimensõess antes referidas. Complementarmente, o recurso a inventários que avaliem crenças, estilos e práticas educativas, bem como a utilização de alguns testes psicológicos revela-se igualmente útil para examinar dimensões da personalidade (e.g., Sixteen Personality Factor Questionnaire -16PF), sintomas psicopatológicos (e.g., Brief Symptom Inventory - BSI), risco de violência (e.g., Spousal Assault Risk Assessment Guide - SARA), sempre na justa medida da complexidade dos casos em análise e de forma intercalada com outras técnicas que estejam pensadas para serem usadas. O princípio fundamental a estabelecer na avaliação é o bem-estar e o superior interesse da criança, devendo realizar-se a avaliação pericial segundo as orientações explanadas e internacionalmente aceites (American Psychological Association, 2009).

\section{Considerações Finais}

A vivência para a criança de conflitos interparentais é uma das experiências mais marcan- tes com implicações sérias no seu ajustamento global. Esses conflitos são vividos de forma tão intensa que muitas crianças chegam a desenvolver comportamentos desadaptativos que podem persistir a curto, médio e longo prazos. Há uma série de fatores que devem ser considerados na análise desse impacto, que em casos pode não ser claramente evidenciado, até porque na avaliação que é feita temos de atender não apenas com o estressor evidente que é o conflito interparental, mas outros fatores associados à criança, à família ou ao contexto. Estes fatores podem funcionar não apenas para aumentar o risco, mas alguns deles como mediadores protetivos de um impacto mais negativo.

O perito que realiza a avaliação psicológica forense em casos de conflitos interparentais deve reunir a análise compreensiva destes aspectos teóricos, bem como munir-se de uma competência técnica e científica que responda ao objetivo de assessoria para que foi chamado. Há uma série de obstáculos e complexidades a serem processadas no âmbito da realização de perícias em casos de famílias que apresentam vivências diversas de conflitualidade, onde muitas vezes existem crianças. Embora não haja universalidade na definição do que possa ser uma parentalidade adequada, este é um dos aspectos sobre o qual nos devemos debruçar quando procedemos estas avaliações. Outros aspectos como as dinâmicas, as relações e vinculações, os afetos e desafetos exprimidos fazem parte do rol de dimensões a analisar, para os quais existem subjacentes processos simples e complexos e que devem ser apurados. A perícia psicológica forense deve ajudar a determinar o que poderá em termos de decisão judiciária refletir o superior interesse da criança.

\section{Referências}

Achenbach, T. M., \& Rescorla, L. A. (2004). The Achenbach System of Empirically Based Assessment (ASEBA). In M. E. Maruish (Eds.), The use of psychological testing for treatment planning and outcomes assessment. Vol. 2: Instruments for Children and Adolescents (3. ed., pp. 179-213). Mahwah, NJ: Lawrence Erlbaum Associates. 
Achenbach, T., Rescorla, L. A., Dias, P., Ramalho, V., Lima, V., Machado, B., \& Gonçalves, M. (2014). Manual do Sistema de Avaliação Empiricamente Validado (ASEBA): Um sistema integrado de avaliação com múltiplos informadores. Manual do periodo pré-escolar e do periodo escolar. Braga, Portugal: Psiquilibrios.

Agulhas, R. (2014). A avaliação das competências parentais e a audição de crianças em contexto judiciário. In Centro de Estudos Judiciários (Ed.), A tutela cível do superior interesse da criança. Vol. III (pp. 224-229) Lisboa: Centro de Estudos Judiciários. Recuperado em http:/www.cej. mj.pt/cej/recursos/ebooks/familia/Tutela_Civel_Superior_Interesse_Crianca_TomoIII.pdf

Agulhas, R., \& Anciães, A. (2014). Casos práticos em Psicologia Forense. Enquadramento legal e avaliação pericial. Lisboa: Edições Sílabo.

Almeida, T., \& Sani, A. (2014). Violência entre pais: Efeitos e transmissão entre gerações. In F. Almeida \& M. Paulino (Eds.), Psicologia, Justiça e Ciências Forenses (pp. 135-150). Lisboa: Pactor.

American Psychological Association. (2009). Guidelines for child custody evaluations in family legal proceedings. Washington, DC: Autor. Recuperado em https://www.apa.org/practice/guidelines/child-custody.pdf

Bevan, E., \& Higgins, D. J. (2002). Is domestic violence learned? The contribution of five forms of child maltreatment to men's violence and adjustment. Journal of Family Violence, 17(3), 223245.

Black, D. S., Sussman, S., \& Unger, J. B. (2010). A further look at the intergenerational transmission of violence: Witnessing interparental violence in emerging adulthood. Journal of Interpersonal Violence, 25(6), 1022-1042.

Bowen, E. (2015). The impact of intimate partner violence on preschool children's peer problems: An analysis of risk and protective factors. Child Abuse \& Neglect, 50, 141-150.

Camisasca, E., Miragoli, S., \& Di Blasio, P. (2016). Families with distinct levels of marital conflict and child adjustment: Which role for maternal and paternal stress? Journal of Child and Family Studies, 25(3), 733-745.

Costa, V. A., \& Sani, A. I. (2007). Sintomatologia de pós-stress traumático em crianças expostas a violência interparental: Do conflito ao ajus- tamento. Revista da Faculdade de Ciências da Saúde, Universidade Fernando Pessoa, 4, 282290. Recuperado em http://bdigital.ufp.pt/handle/10284/419.

Cottrell, B., \& Monk, P. (2004). Adolescent-to-parent abuse: A qualitative overview of common themes. Journal of Family Issues, 25, 1072-1095.

Coutinho, M. J., \& Sani, A. I. (2008). Evidência empírica na abordagem sobre as consequências da exposição à violência interparental. Revista $d a$ Faculdade de Ciências da Saúde, Universidade Fernando Pessoa, 5, 284-293. Recuperado em https://bdigital.ufp.pt/dspace/handle/10284/970.

Cox, J., Paley, B., \& Harter, K. (2001). Interparental conflict and parent - Child relationships. In J. H. Grych \& F. D. Fincham (Eds.), Interparental conflict and child development (pp. 249-272). Cambridge, MA: Cambridge Press.

Cunningham, A., \& Baker, L. (2004). What about me! Seeking to understand the child's view of violence in the family. London: Centre for Children and Families in the Justice System.

Dankoski, M. E., Keiley, M. K., Thomas, V., Choice, P., Lloyd, S. A., \& Seery, B. L. (2006). Affect regulation and the cycle of violence against women: New directions for understanding the process. Journal of Family Violence, 21, 327-339.

Dunn, J., \& Davies, L. (2001). Sibling relationships and interparental conflict. In J. H. Grych \& F. D. Fincham (Eds.), Interparental conflict and child development (pp. 273-290). Cambridge, MA: Cambridge Press.

English, J. D., Graham, J. C., Newton, R. R., Lewis, T. L., Thompson, R., Kotch, J. B., \& Weisbart, C. (2009). At-risk and maltreated children exposed to intimate partner aggression/violence What the conflict looks like and its relationship to child outcomes. Child Maltreatment, 14(2), 157-171.

Fergusson, D. M., Boden, J. M., \& Horwood, L. J. (2008). Developmental antecedents of interpartner violence in a New Zealand birth cohort. Journal of Family Violence, 23, 737-753.

Fialho, A. J. (2012). Guia prático do divórcio e responsabilidades parentais. Coleção Guias Práti$\cos$ (2. ed.). Lisboa: Centro de Estudos Judiciários. Recuperado em http://www.cej.mj.pt/cej/ recursos/ebooks/familia/guia_pratico_divorcio_ responsabilidades_parentais.pdf 
Gomes de Sousa, J. H. (2011). A 'perícia' técnica ou científica revisitada numa visão prático-judicial. Julgar, 15, 27- 52.

Herrenhohl, T., Sousa, C, Tajima, E., Herrenhohl, R., \& Moylan, C. (2008). Intersection of child abuse and children's exposure to domestic violence. Trauma, Violence \& Abuse, 9, 84-99.

Howell, K. H. (2011). Resilience and psychopathology in children exposed to family violence. $\mathrm{Ag}$ gression and Violent Behavior, 16, 562-569.

Hughes, H. M., Graham-Bermann, S. A., \& Gruber, G. (2001). Resilience in children exposed to domestic violence. In S. A. Graham-Bermann \& J. Edleson (Eds.), Domestic violence in the lives of children. The future of research, intervention and social policy (pp. 67-90). Washington, DC: American Psychological Association.

Huss, M. T. (2011). Psicologia Forense. Pesquisa, prática clínica e aplicações. Porto Alegre, RS: Artmed.

Laporte, L., Jiang, D., Pepler, D. J., \& Chamberland, C. (2011). The relationship between adolescents' experiences of family violence and dating violence. Youth and Society, 43, 3-27.

Levendosky, A. A., Bogat, G. A., \& Huth-Bocks, A. C. (2011). The influence of domestic violence on the development of the attachment relationship between mother and young child. Psychoanalytic Psychology, 28, 512-527.

Machado, M. L., \& Sani, A. (2014). Avaliação psicológica forense na regulação do exercício das responsabilidades parentais. Perceções dos juízes. In F. Almeida \& M. Paulino (Eds.), Psicologia, Justiça e Ciências Forenses (pp. 357-371). Lisboa: Pactor.

Machado, M. L. \& Sani, A. (2015). A (síndroma de) alienação parental. Revista Digital Lusobrasileira de Alienação Parental, 7, 164-176. Recuperado em http://issuu.com/sandraines3/docs/ revista_aliena o_parental_7.

Manning, L. G., Davies, P. T., \& Cicchetti, D. (2014). Interparental violence and childhood adjustment: How and why maternal sensitivity is a protective factor. Child Development, 85(6), 2263-2278.

Marasca, A. R., Colossi, P. M., \& Falcke, D. (2013). Violência conjugal e família de origem: Uma revisão sistemática da literatura de 2006 a 2011. Temas em Psicologia, 21(1), 221-243.
Molinari, F., \& Sani, A. I. (2015a). Mediação Familiar em contextos de alienação parental: Perspectiva para uma coparentalidade positiva. In C. P. Rosa \& L. M. B. Thomé (Eds.), Um presente para construir o futuro: Escritos sobre Família e Sucessões (pp. 184-209). Porto Alegre, RS: Instituto Brasileiro de Direito de Família - Seção do Rio Grande do Sul.

Molinari, F., \& Sani, A. I. (2015b). Um olhar multidisciplinar sobre o fenômeno da Alienação Parental. In C. P. Rosa (Ed.), Família e sucessões: Novos temas e discussões (pp. 326-338). Porto Alegre, RS: RJR.

Molinari, F., \& Trindade, J. (2014). Reflexões sobre alienação parental e a escala de indicadores legais de alienação parental. In C. P. Rosa \& L. M. B. Thomé (Eds.), O Direito no lado esquerdo do peito: Ensaios sobre direito de família e sucessões (pp. 23-33). Porto Alegre, RS: Instituto Brasileiro de Direito de Família - Seção do Rio Grande do Sul.

Patias, N. D., Bossi, T. J., \& Dell’Aglio, D. D. (2014). Repercussões da exposição à violência conjugal nas características emocionais dos filhos: Revisão sistemática da literatura. Temas em Psicologia, 22(4), 901-915.

Peixoto, C., Ribeiro, C., \& Manita, C. (2007). Avaliação psicológica forense das capacidades parentais. Revista da SPTM, 11(2), 142-156.

Pereira, A., \& Matos, M. (2011). Avaliação psicológica das responsabilidades parentais nos casos de separação e divórcio. In M. Matos, R. A. Gonçalves, \& C. Machado (Eds.), Manual de Psicologia Forense: Contextos, práticas e desafios (pp. 311-347). Braga, Portugal: Psiquilíbrios.

Pereira, D., \& Alarcão, M. (2010). Avaliação da parentalidade no quadro da proteção à infância. Temas em Psicologia, 18(2), 499-517.

Pryor, J. E., \& Pattison, R. (2007). Adolescents' perceptions of parental conflict: The downside of silence. Journal of Family Studies, 13(1), 72-77.

Sani, A. I. (2004a). O discurso de crianças expostas à violência interparental - Estudo Qualitativo. Psychologica, 36, 109-130.

Sani, A. I. (2004b). Abordagens teóricas da violência interparental: Compreensão do ajustamento da criança ao conflito dos pais. Psicologia: Teoria, Investigação e Prática, 2, 153-177. 
Sani, A. I. (2006). As variáveis mediadoras do impacto na criança da exposição à violência interparental. Psicologia: Teoria, Investigação e Prática, 11(2), 111-133.

Sani, A. I. (2007). Las consecuencias de la violencia interparental en la infancia. In R. Arce, F. Fariña, E. Alfaro, C. Civera, \& F. Tortosa (Eds.), Psicología Jurídica: violencia y victimas (pp. 13-21). Valencia, Espanha: Sociedad Española de Psicología y Ley.

Sani, A. I. (2011). Crianças vitimas de violência: Representações e impacto do fenómeno. Porto, Portugal: Edições Universidade Fernando Pessoa.

Sani, A., \& Almeida, T. (2011a). Violência interparental: A vitimação indirecta de crianças. In A. I. Sani (Ed.), Temas de Vitimologia: Realidades emergentes e respostas sociais (pp. 11-31) Coimbra, Portugal: Almedina.
Sani, A., \& Almeida, T. (2011b). Avaliação psicológica de crianças expostas à violência interparental. In M. Matos, R. A. Gonçalves, \& C. Machado (Eds.), Manual de Psicologia Forense: Contextos, práticas e desafios (pp. 159-173). Braga, Portugal: Psiquilibrios.

Simon, V. A., \& Furman, W. (2010). Interparental conflict and adolescents' romantic relationship conflict. Journal of Research on Adolescence, 20(1), 188-209.
Recebido: $16 / 11 / 2015$

$1^{a}$ revisão: $25 / 02 / 2016$

Aceite final: 04/03/2016 